\title{
Analysis and prediction of human acetylation using a cascade classifier based on support vector machine
}

\author{
Qiao Ning, Miao Yu, Jinchao Ji, Zhiqiang $\mathrm{Ma}^{*}$ and Xiaowei Zhao ${ }^{*}$ (D)
}

\begin{abstract}
Background: Acetylation on lysine is a widespread post-translational modification which is reversible and plays a crucial role in some biological activities. To better understand the mechanism, it is necessary to identify acetylation sites in proteins accurately. Computational methods are popular because they are more convenient and faster than experimental methods. In this study, we proposed a new computational method to predict acetylation sites in human by combining sequence features and structural features including physicochemical property (PCP), position specific score matrix (PSSM), auto covariation (AC), residue composition (RC), secondary structure (SS) and accessible surface area (ASA), which can well characterize the information of acetylated lysine sites. Besides, a two-step feature selection was applied, which combined mRMR and IFS. It finally trained a cascade classifier based on SVM, which successfully solved the imbalance between positive samples and negative samples and covered all negative sample information.

Results: The performance of this method is measured with a specificity of $72.19 \%$ and a sensibility of $76.71 \%$ on independent dataset which shows that a cascade SVM classifier outperforms single SVM classifier.

Conclusions: In addition to the analysis of experimental results, we also made a systematic and comprehensive analysis of the acetylation data.

Keywords: Lysine, Acetylation sites, Human, Support vector machine, Cascade classifier, Sequence features, Structural feature, Systematic and comprehensive analysis
\end{abstract}

\section{Key points}

1. Specifically predict acetylated lysine sites in human.

2. Combine sequence features and structural features to translate proteins into numerical vector.

3. Build a cascade classifier based on support vector machine.

4. Solve the imbalance between positive samples and negatives, and cover all negative sample information.

\section{Background}

Protein acetylation is the process of adding acetyl groups $\left(\mathrm{CH}_{3} \mathrm{CO}-\right)$ to lysine residues on protein chain. As a widespread type of protein post-translational modifications (PTMs), acetylation on lysine plays a significant role in various organisms. In eukaryotes, the function of

\footnotetext{
* Correspondence: zhaoxw303@nenu.edu.cn; zhaoxw303@nenu.edu.cn School of Information Science and Technology, Northeast Normal University, Changchun 130117, China
}

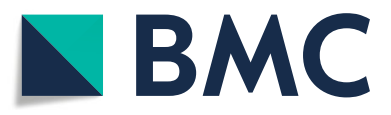

(c) The Author(s). 2019 Open Access This article is distributed under the terms of the Creative Commons Attribution 4.0 International License (http://creativecommons.org/licenses/by/4.0/), which permits unrestricted use, distribution, and reproduction in any medium, provided you give appropriate credit to the original author(s) and the source, provide a link to the Creative Commons license, and indicate if changes were made. The Creative Commons Public Domain Dedication waiver (http://creativecommons.org/publicdomain/zero/1.0/) applies to the data made available in this article, unless otherwise stated. acetylation is mainly focused on the influence of cell chromosome structure and the activation of nuclear transcription factors. However, the recent study of the flux of proteins and the metabolic pathway of different species revealed that a large number of non-nuclear proteins were acetylated in the metabolic pathway which would provide an important basis for the use of various drugs or vitamins in real life. In prokaryotes, protein acetylation is mainly manifested in the following aspects: directly effecting the enzyme activity, affecting the interaction between proteins, influencing the metabolic flow.

Though acetylation is very common in biological process, knowledge of lysine acetylation is still quite limited. Since it is extremely important to understand the molecular mechanism of acetylation in biological systems by identifying acetylated substrate proteins along with acetylation sites, more and more focus is put on this field. Compared with the labor-intensive and time-consuming traditional 
experimental methods, such as liquid chromatographymass spectrometry, high performance liquid chromatography assays and spectrophotometric assays [1, 2], computational approaches of acetylation sites are much more popular because of their convenience and fast speed. Recent years, many computational classifiers have been built to identify PTM sites through various types of two-class machine learning algorithms. In 2014, Lu et al. used MDDlogo to cluster positive samples and built a series of classifiers using several kinds of sequence features [3]. Deng et al. proposed a classifier called GPS-PAIL to predict HAT-specific acetylation sites for up to seven HATs, including CREBBP, EP300, HAT1, KAT2A, KAT2B, KAT5 and KAT8 [4]. There are at least a dozen of additional computational programs developed in earlier studies for the prediction of lysine acetylation sites, such as AceK, ASEB, BPBPHKA, EnsemblePail, iPTMmLys, KAcePred, KA-predictor, LAceP, LysAcet, NAce, PLMLA, PSKAcePred and SSPKA [5-17].

However, these classifiers didn't give a good solution of the imbalance between positive and negative samples. Besides, post-translational modification of proteins is species-specific, which means that different methods should be considered for the prediction of PTM sites in different organisms. Therefore, in this study, we developed a method specific to human using a cascade classifier of support vector machine to solve the imbalance problem of positive and negative samples combined with both sequence and structural feature descriptors. Finally, we made a systematic and comprehensive analysis of human acetylation data and the prediction results. The flow chart of our method is shown in Fig. 1.

\section{Methods}

\section{Dataset}

In this study, acetylated protein data were derived from CPLM [18], PLMD [19], PhosphoSitePlus [20], UniprotKB/Swiss-prot [21] and RCSB database [22] according to following five steps.

Step 1. First of all, we downloaded all the human acetylated protein sequences from CPLM, PLMD, PhosphoSitePlus and UniprotKB/Swiss-prot (10,146 proteins).

Step 2. Secondly, we removed proteins using CD-HIT with identity of $40 \%$. 6834 protein sequences were left and labeled as D1.

Step 3. Next, all PDB sequences were downloaded from RCSB database and were labeled as D2.

Step 4. Then, PSI-BLAST was applied to calculate the similarity between D1 and D2. And each protein sequence in D1 only retained one matching result that had the highest score. Proteins in D1 that have no matching result were excluded.

Step 5. Finally, PDB files of proteins in D1, that were validated by X-ray diffraction and resolution less than 2.0 $\AA$, were download from RCSB database.

After these five steps, we obtained 1213 proteins which have 3D structural information, from which 243 proteins including 451 acetylation sites and 4918 non-acetylation sites were regarded as validation dataset (used for parameter optimization and feature selection), and the rest 970 proteins including 1956 acetylation sites and 18,061 non-acetylation sites were regarded as the training dataset. To evaluate the performance of our method, we downloaded acetylated

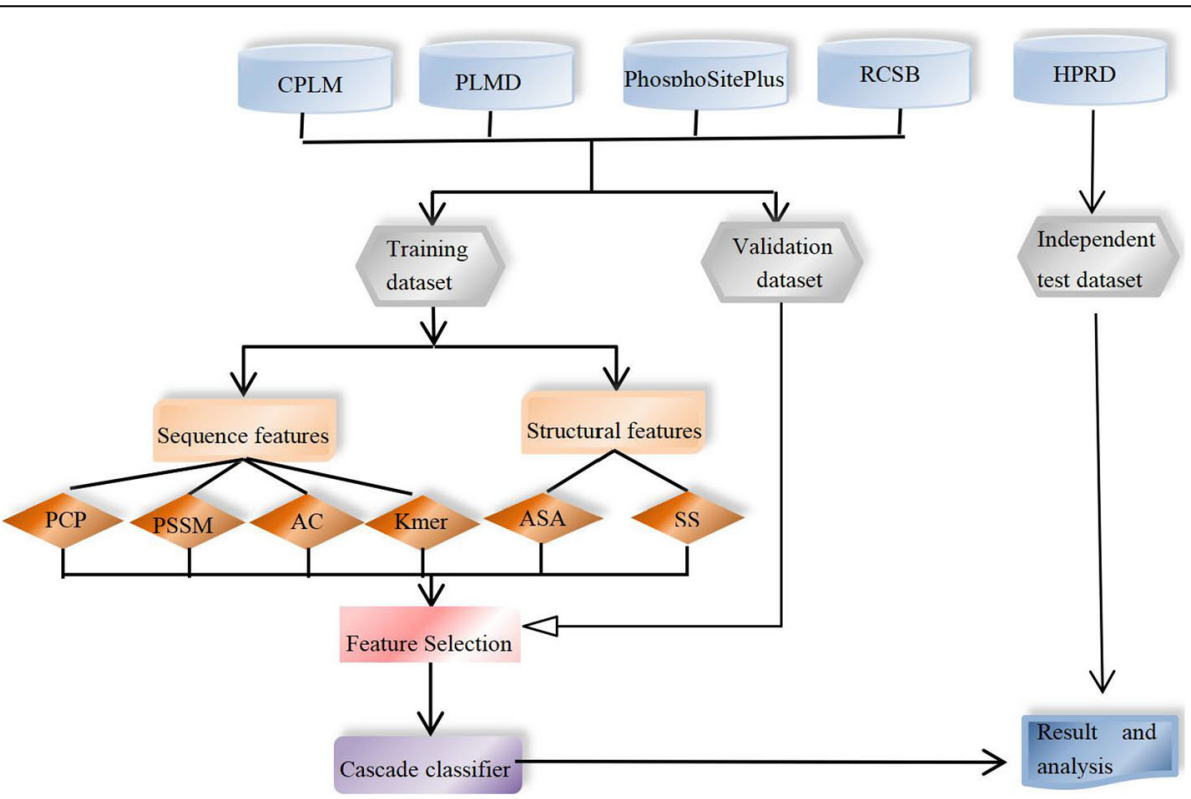

Fig. 1 The flow chart of this method 
data from HPRD [23] as independent test data, in which proteins that have greater than $40 \%$ identity with training data are excluded.

Subsequently, similar to the development of other PTM site predictors $[24,25]$, the sliding window strategy was utilized to extract samples. A window size of 19 was adopted in this paper with 9 residues located upstream and 9 residues located downstream of the lysine sites in the protein sequence and ' $\mathrm{X}$ ' was used when the number of residues downstream or upstream is less than 9 .

\section{Features}

To develop an accurate tool to predict protein acetylation sites, it is necessary and important to translate proteins into numerical vector with comprehensive and proper features. Diverse kinds of features represent different information of protein. In this study, we tested variety sequence features and structural features including physicochemical property (PCP), position specific score matrices (PSSM), auto covariation $(\mathrm{AC})$, residue composition (RC), secondary structure (SS) and accessible surface area (ASA).

\section{Physicochemical property (PCP)}

AAindex is a database which includes amino acid mutation matrices and amino acid indices [26]. Removing 13 PCPs that include the value "NA", 531 PCPs are available. An amino acid index is a set of 20 numerical values on behalf of the specificity and diversity of structure and function of amino acids. PCPs have ever been successfully used to predict many protein modifications in previous papers, such as S-glutathionylation and acetylation [27]. Character ' $\mathrm{X}$ ' was represented by ' 0 ' in each kind of physicochemical property. For each physicochemical property, we built a classifier based on it, and test its performance with validation data. Finally, we chose four kinds of physicochemical properties that have the best performances (compareing their Matthew's correlation coefficient value), activation gibbs energy of unfolding, $\mathrm{pH} 7.0$ [28], activation gibbs energy of unfolding, $\mathrm{pH} 9.0$ [28], normalized flexibility parameters (B-values) for each residue surrounded by one rigid neighbours [29], averaged turn propensities in a transmembrane helix [30].

\section{Position specific scoring matrices (PSSM)}

The evolutionary conservation is one of the most important aspects in biological analysis, and residues with stronger conservation may be more important for protein function. PSI-BLAST [31] is a tool to calculate the conservation state of specific residues. In this work, we used PSI-BLAST against the swissprot protein database to calculate position specific scoring matrices (PSSM), which is a kind of feature that regarding the evolutionary conservation of a protein.
PSSM has been widely used in some other prediction problems [32-35] and obtained satisfactory results. In PSSM, each residue in peptide had 20 conservative states against 20 different amino acids, so we can get $380(=19 * 20)$ dimension features.

\section{Auto covariation (AC)}

There are many interactions between amino acids in proteins, and the physicochemical properties of proteins can reflect these interactions. Auto convariation variable [36, 37] represents the correlation of the same property between two residues separated by a fixed value, that we called lag, which means the distance between two sites. Here, proteins are replaced by four kind of physicochemical properties which we mentioned in chapter 2.2.1. The calculation formula of $\mathrm{AC}$ value is as follows.

$$
X_{i, j}=\frac{p_{i, j}-p_{j}}{S_{j}}
$$

First, normalize physicochemical properties to zero mean and unit standard deviation (SD) according to:

in which $j$ means different physicochemical properties, $P_{i, j}$ is the $j$-th descriptor value for $i$-th amino acid, $P_{j}$ is the mean of $j$-th descriptor over the 20 amino acids and $\mathrm{S}_{\mathrm{j}}$ is the corresponding SD. Then,

$$
A C_{\mathrm{lg}, j}=\frac{1}{n-\lg } \sum_{i=1}^{n-\lg }\left(X_{i, j}-\frac{1}{n} \sum_{i=1}^{n} X_{i, j}\right) \times\left(X_{(i+\lg ), j}-\frac{1}{n} \sum_{i=1}^{n} X_{i, j}\right)
$$

Where $i$ is the position of protein sequence, $j$ is one of the residues, $\mathrm{n}$ is the size of the window, $\lg$ is the value of lag. We have chosen two lag values, 1 and 2 .

\section{Residue composition ( $R C)$}

Residue composition [38] represents the occurrence frequencies of different amino acid pairs in one subsequence. It is a good representation of the local composition of protein sequences. In this work, the dimension of residue composition is 20 . The matrix includes the frequencies of 20 amino acids ("A", " $C$ ",

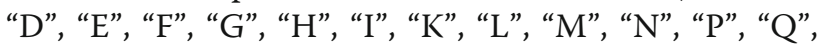
"R", “S”, “T", "V", “W", "Y").

\section{Secondary structure (SS)}

Protein secondary structure reflects the function of protein and impacts many kind of protein reactions [39]. Secondary structure includes alpha helix, beta bridge, strand, helix-3, helix-5, turn and bend. DSSP is a powerful tool to compute the secondary structure for each residue. DSSP [40] gives "H", "B", "E", "G", "I", “T" and " $\mathrm{S}$ " as output which indicate alpha helix, beta bridge, strand, helix-3, helix-5, turn and bend. In this 
work, “0000001", “0000010", “0000100", “0001000", "0010000", "0100000", "1,000,000" stand for "H", "B", "E", "G", "I", "T" and "S", respectively, and "X" is represented by " 0000000 ".

\section{Accessible surface area (ASA)}

As a key property of amino acid sites, accessibility surface area plays a crucial part in protein function [41] because biological reaction always happens on the surface of proteins. Values of the accessible surface area (ASA) for residues from PDB were calculated using the surface_racer_5.0 with the $1.4 \AA$ rolling probe.

\section{Performance assessment}

Four intuitive evaluation indexes were derived from Chou's symbols introduced for studying protein signal peptides [42], and they have been successfully used in some papers [43-49]. Thus, we utilized these four indexes to evaluate the proposed predictor: sensitivity $(\mathrm{Sn})$, specificity (Sp), accuracy (Acc), Matthew's correlation coefficient (MCC). And the four measurements are defined as following:

$$
\begin{aligned}
& S n=\frac{T P}{T P+F N} \\
& S n=\frac{T P}{T P+F P}
\end{aligned}
$$

$$
A c c=\frac{T P+T N}{T P+T N+F P+F N}
$$

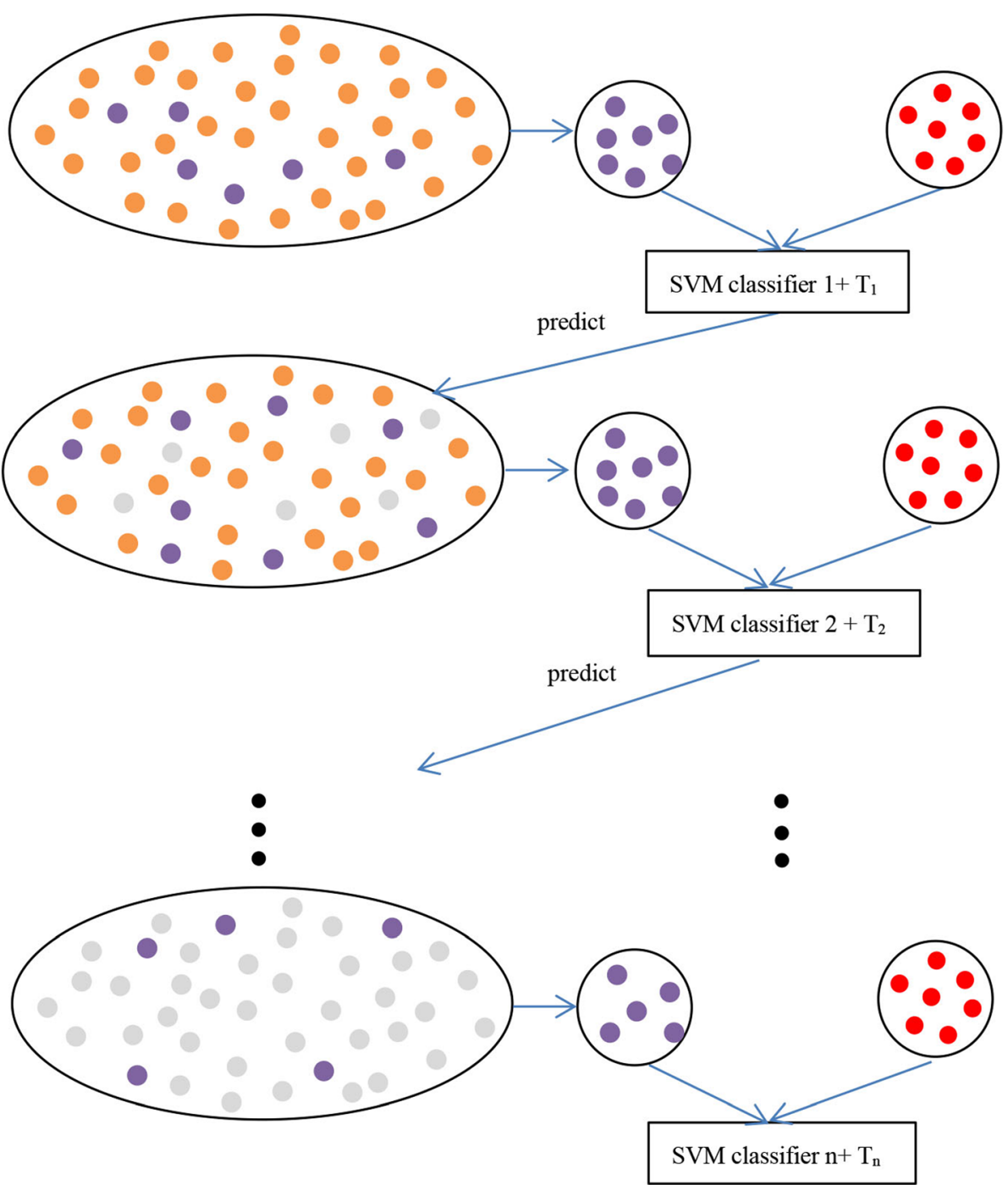

Fig. 2 The process of cascade SVMs. Red dots are positive samples. Orange dots are non-acetylation samples. Purple dots are selected negative samples. Grey dots are non-acetylation samples that are correctly predicted and deleted 
Table 1 Comparison between sequence features and combination features (sequence and structural features)

\begin{tabular}{lllll}
\hline & $\mathrm{Sn}(\%)$ & $\mathrm{Sp}(\%)$ & $\mathrm{Acc}(\%)$ & $\mathrm{MCC}$ \\
\hline Sequence features (PCP + PSSM+AC+RC) & 70.66 & 62.15 & 66.41 & 0.119 \\
Sequence and structural features & 76.71 & 72.19 & 74.45 & 0.19 \\
(PCP + PSSM+AC + RC + SS + ASA) & & & & \\
\hline
\end{tabular}

$$
M C C=\frac{T P \times T N-F P \times F N}{\sqrt{(T P+F N) \times(T N+F P) \times(T P+F P) \times(T N+F N)}}
$$

where $T P$ and $T N$ mean the number of truely identified acetylation sites and non-acetylation sites. $F N$ is the number of the acetylation sites incorrectly predicted as non-acetylation sites, and FP represents the number of non-acetylation sites incorrectly predicted as acetylation sites.

\section{Feature selection scheme}

Varied features are often redundant and some features are noisy and lead to negative impacts, so it is necessary to remove the irrelevant and redundant features from original feature set using an efficient feature selection method. In this study, we performed a two-step feature selection method to select the optimal feature subsets. After comparison among different evaluation index, we find that mRMR (maximum relevance and minimum redundancy) [50] can give the best result for feature selection. The detailed steps of feature selection method are as follows:

1) For the first step, mRMR value was calculated to estimate the relevance and redundancy between features. Then, we ranked these features based on mRMR value, and picked out the top 300 features.

2) Secondly, features in ranked list were added one by one into feature subset, and we built models on these feature subsets.

3) Then, validation dataset was used to evaluate the performance of these feature subsets.

4) In the end, the feature subset that has the best performance was the optimal feature subset.

In this study, we regarded MCC value as the evaluation performance in feature selection because MCC value is a comprehensive evaluation index for positive and negative samples.

\section{Cascade classifier}

Support vector machine (SVM) is a widely used machine learning algorithm based on statistical learning theory [51]. For actual implementation, LIBSVM package (version 3.0) [52] with radial basis kernels (RBF) is used, where the kernel width parameter $\gamma$ represents how the samples are transformed to a high dimensional space.
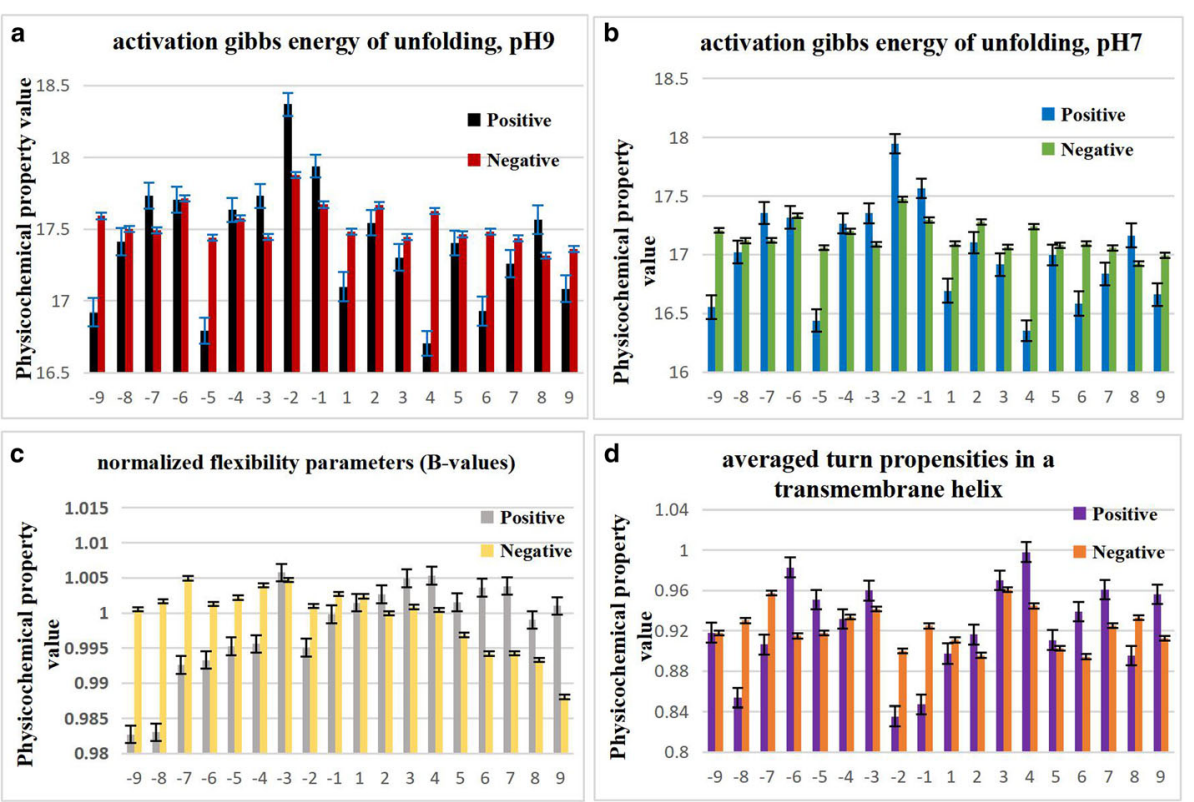

Fig. 3 The average values of four physicochemical properties around the center residue in positive dataset and negative dataset, respectively. (a) is for activation gibbs energy of unfolding, $\mathrm{pH}$, (b) is for activation gibbs energy of unfolding, $\mathrm{pH}$, (c) is for normalized flexibility parameters(Bvalues), and (d) is for averaged turn propensities in a transmembrane helix 


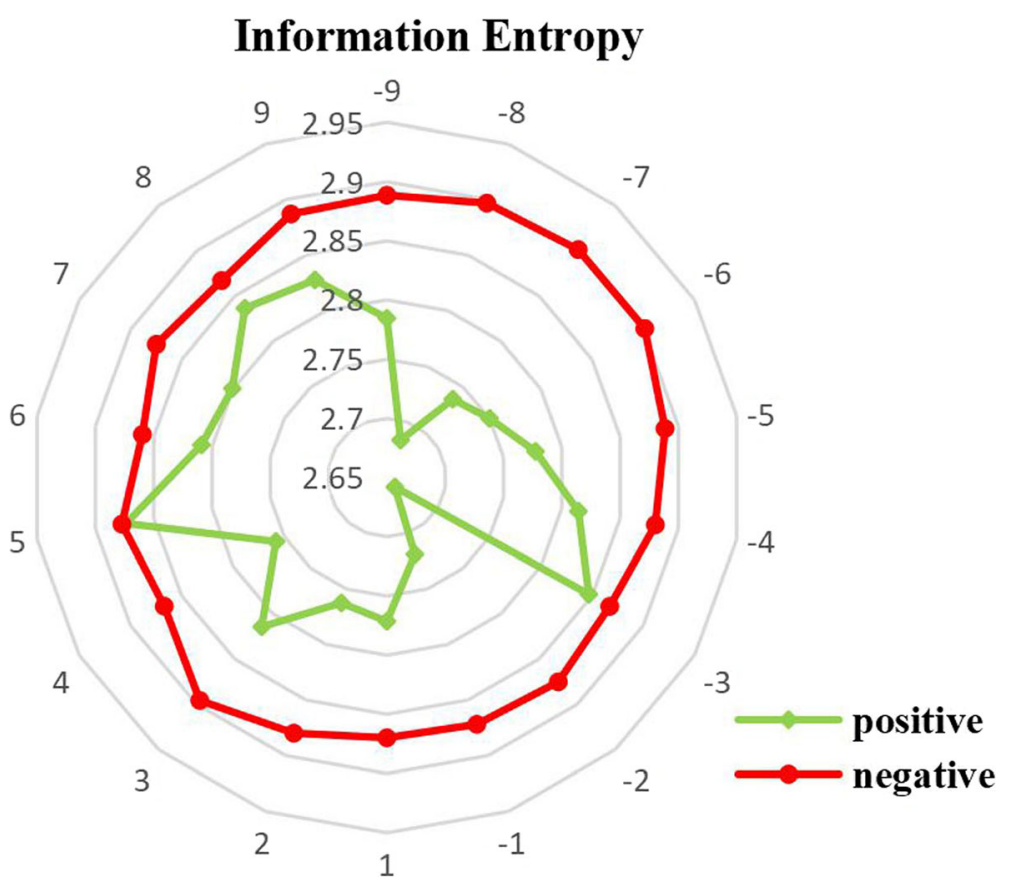

Fig. 4 Comparison of conservation in each position between acetylated and non-acetylated peptides by information entropy values

However, traditional SVM also suffer from the problem of imbalance training dataset. If all the nonacetylation sites are regarded as negative samples, the prediction results will be biased towards the negative samples and the accuracy is greatly reduced. Enlightened by the method proposed in Wei's work [53], we built a cascade classifier based on SVM to predict acetylation sites. Figure 2 shows the process of the cascade SVMs and following is the step of building this classifier, in which PD represents positive data, TND represents total negative data and ND represents subset of negative data (the same amount of samples as PD).

Step1. Randomly select a subset of ND from TND and generate a balanced classifier $S_{i}$ with PD and ND.

Step2. Test PD and TND with classifier.
Step3 Sort the decision value of PD from large to small and the $0.95^{*}$ Mth decision value of $\mathrm{PD}$ is regarded as threshold $\mathrm{T}_{\mathrm{i}}$ (M is the number of acetylation samples in PD).

Step4. Non-acetylation samples whose decision value is lower than $T_{i}$ are excluded from TND, and $\left(S_{i}, T_{i}\right)$ form the ith layer of cascade classifier.

Step5. Select non-avetylation sites from TND that have lower decision value as new ND, and generate a new classifier $\mathrm{S}_{\mathrm{i}+1}$ with PD and ND.

Step6. Repeat Step2-5 until less than 0.05*18061(the number of original TND) can be removed from TND.

$0.95 *$ Mth decision value of PD as threshold means that we allow 0.05 times positive samples to be predicted incorrectly in each round. In this case, if less than 0.05 times negative samples can be correctly predicted, the

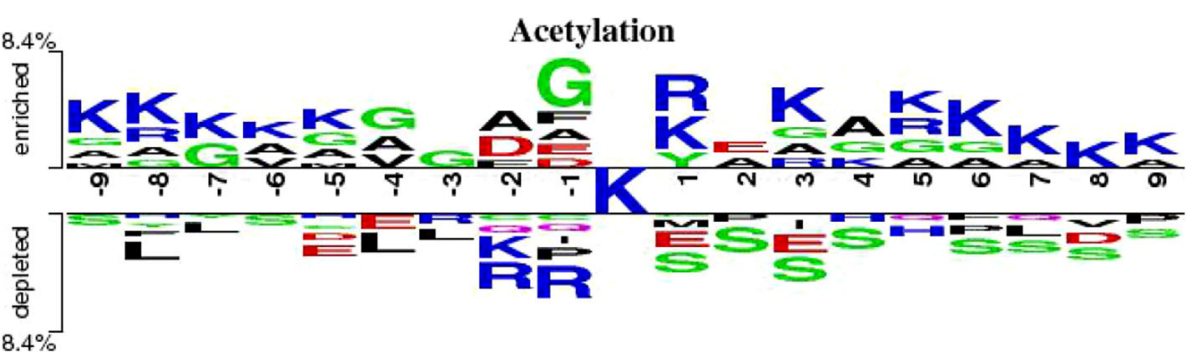

Fig. 5 Two sample logos of the compositional biases around acetylation sites compared to non-acetylation sites. Statistically significant symbols are plotted using the size of the symbol that is proportional to the difference between the two samples. Residues are separated in two groups: (i) enriched in the positive sample, and (ii) depleted in the positive sample. Color of symbols depends on the polarity of the side chain groups in corresponding amino acids 

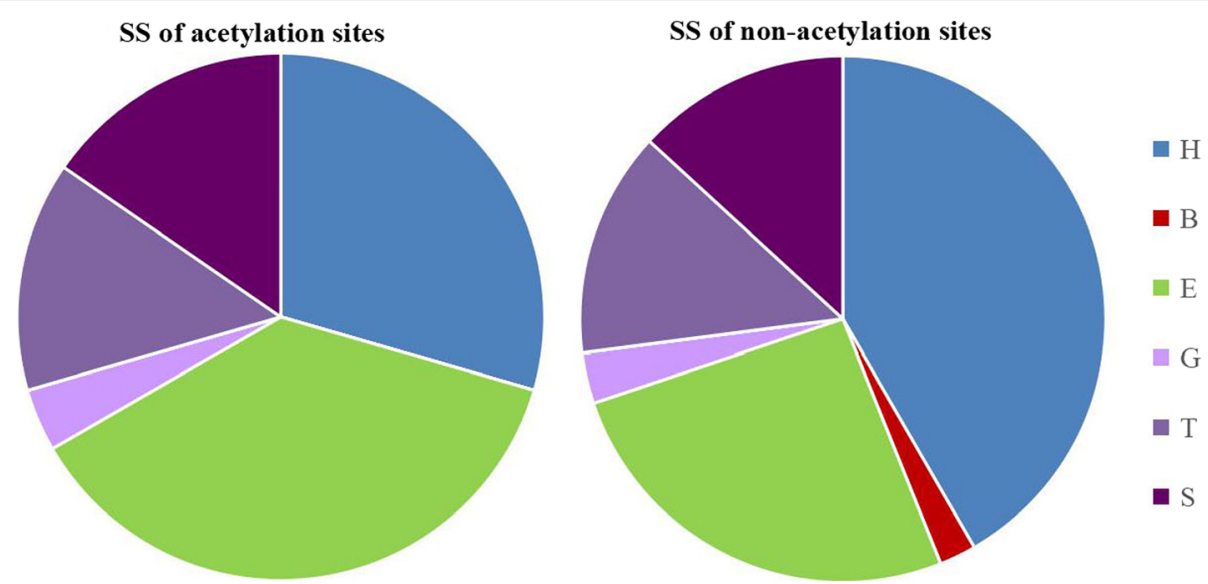

Fig. 6 The frequency of different kinds of secondary structure in acetylation site and non-acetylation site

average value of $\mathrm{Sp}$ and $\mathrm{Sn}$ will be less than 0.5 , then we should stop.

Finally, we get a cascade classifier containing n SVM classifiers, $\left\{\left(\mathrm{S}_{1}, \mathrm{~T}_{1}\right),\left(\mathrm{S}_{2}, \mathrm{~T}_{2}\right), \ldots,\left(\mathrm{S}_{\mathrm{n}}, \mathrm{T}_{\mathrm{n}}\right)\right\}$. For a query sample $q$, it will be predicted from $\left(S_{1}, T_{1}\right)$ to $\left(S_{n}, T_{n}\right)$ orderly. If the sample $\mathrm{q}$ is predicted as the negative sample at any layer $\mathrm{i}, \operatorname{Deci}_{\mathrm{q}}<\mathrm{T}_{\mathrm{i}}$, the prediction will terminate, and $\mathrm{q}$ is classified as non-acetylation site, or it is transferred to $\mathrm{i}+1$ layer for further prediction. It will be classified as acetylation site only if all the SVM classifiers predict it as positive sample.

\section{Results}

\section{Comparison based on features}

To develop an accurate tool to predict protein acetylation sites, it is necessary and important to translate protein with comprehensive and proper features into numerical vector. Sequence features are commonly used in prediction because protein sequences are easily available. However, sometimes sequence information is not enough to describe the characteristic of proteins or amino acids, because proteins are three-dimensional, not only a chain, and the 3D structure is closer to the real conformation of proteins. Structural features are used to depict spatial information of amino acids.

In this study, we tested several features, including sequence features (PCP, PSSM, AC, RC) and structural features (SS, ASA). To verify the importance of structural features, we made a comparison between sequence features and combination features, and the performances are listed in Table 1. Combination features get a higher performance on $\mathrm{Sn}, \mathrm{Sp}$, Acc and MCC than sequence

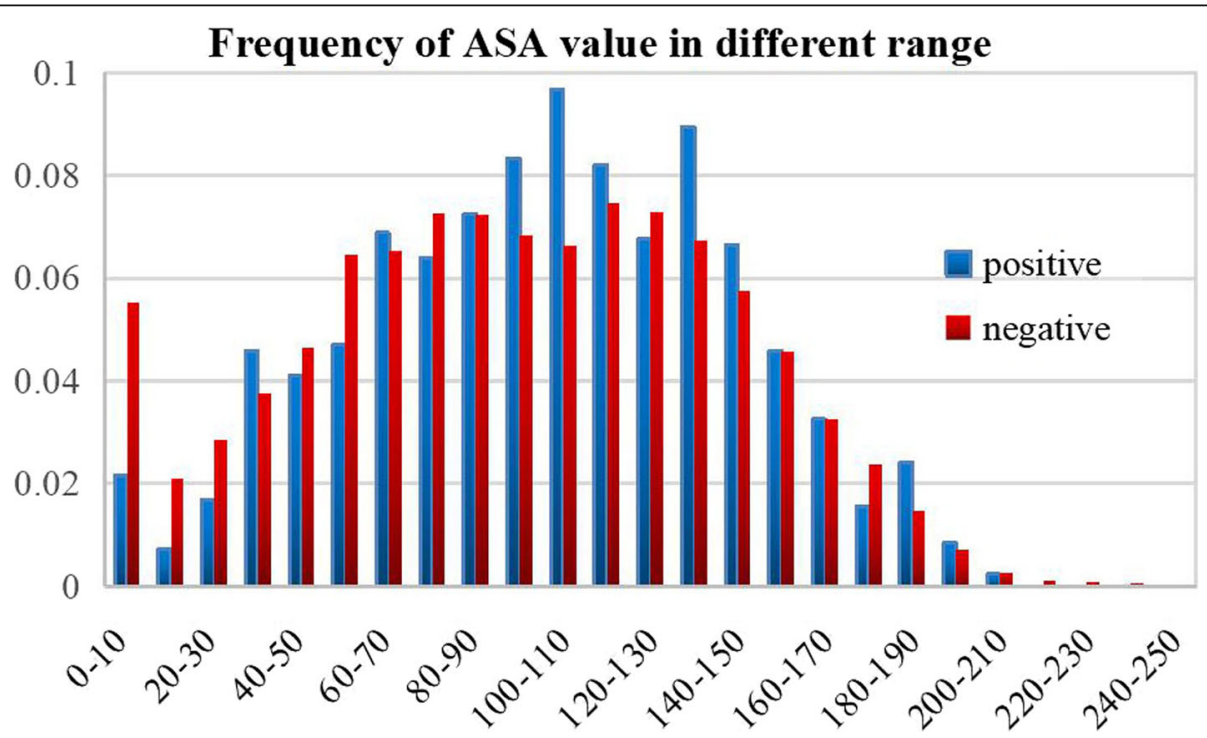

Fig. 7 Comparison of frequency of accessible surface area between acetylation sites and non-acetylation sites 
MCC

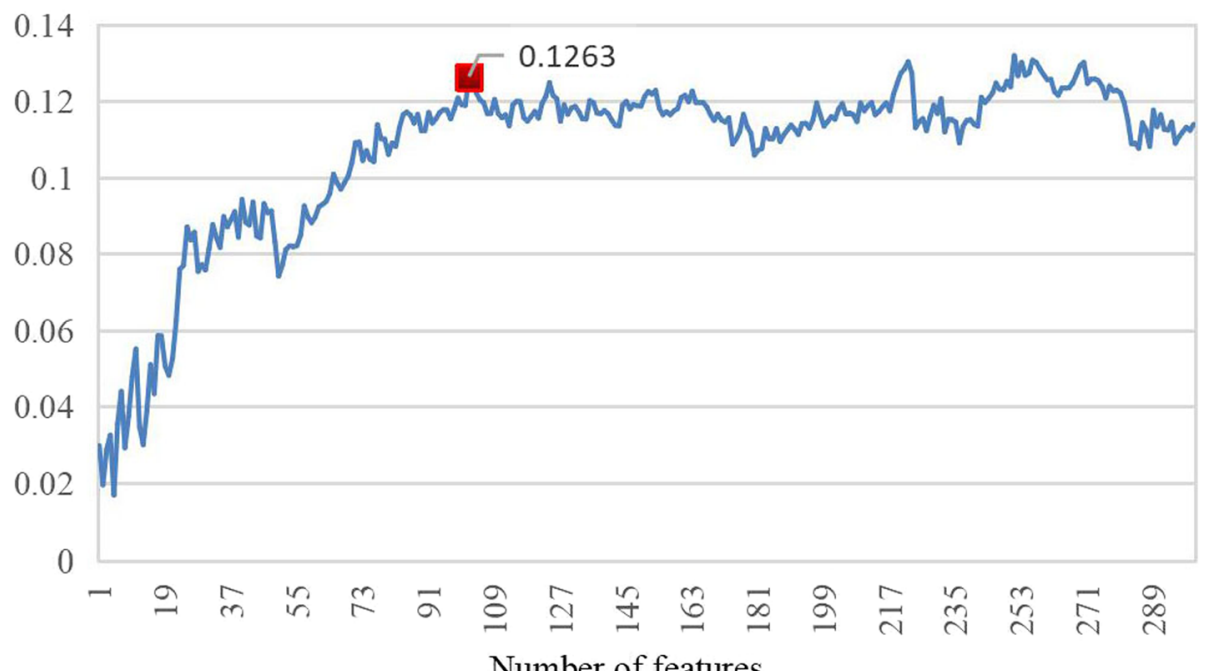

Number of features

Fig. 8 MCC curve of different number of features in final feature set

features, which indicates that structural features is significant and useful in prediction.

\section{Analysis of sequence features}

We calculate the average values and standard errors of four physicochemical properties around the center residue in positive dataset and negative dataset, respectively, and the results are shown in Fig. 3.

As shown in Fig. 3(a)(b)(c)(d), we can see that positions close to the center lysine have distinctly different values of all these four physicochemical properties. Especially in Fig. 3(a) and (b), positions in the upstream and close to lysine residues have greater values in positive dataset than in negative dataset while in the downstream, positive values are weaker. Figure 3(a) and (b) represents the activation gibbs energy of unfolding in $\mathrm{pH} 7.0$ and in $\mathrm{pH} 9.0$, so we can conclude from the above results that acetylation may change the direction of the unfolding process from one side to another side.

The evolution history represents important information of a residue, and evolution information reflects the conservation information because a conserved position is more difficult to be replaced. We calculated the information entropy (IE) of positions in acetylated peptides and

Table 2 Comparison of performance between before feature selection and after feature selection

\begin{tabular}{llllll}
\hline & $\mathrm{Sn}(\%)$ & $\mathrm{Sp}(\%)$ & $\mathrm{Acc}(\%)$ & MCC & Dimension \\
\hline Before feature selection & 63.19 & 52.58 & 57.88 & 0.087 & 632 \\
After feature selection & 69.18 & 53.58 & 61.38 & 0.1263 & 102 \\
\hline
\end{tabular}

non-acetylated peptides, and results are shown in Fig. 4. Comparison between acetylated and non-acetylated peptides indicates that residues around acetylation sites are more conservative than those in the flanking position of non-acetylation sites, especially in the downstream.

Figure 5 shows the distribution of amino acids around center lysine. Figure 5 shows that the distribution of amino acid residues between acetylation and non-acetylation are distinct. In acetylation data, lysine $(K)$ is enriched around acetylated lysine, especially on position 1 . While in non-acetylation data, serine $(S)$ is enriched, especially on position 1, 2, 3 and 4. Thus, it is necessary to utilize frequency-dependent feature, $\mathrm{RC}$, and position-dependent feature, AC, to represent the characteristics of samples.

\section{Analysis of structural features}

We evaluate the frequency of different kinds of secondary structure in acetylation site and non-acetylation site, which is defined as:

$$
F_{i}=\frac{N_{i}}{N}, i=\{H, B, E, G, I, T, S\}
$$

Table 3 Performances of cascade classifier and single SVM classifier

\begin{tabular}{lllll}
\hline & Sn(\%) & Sp(\%) & Acc(\%) & MCC \\
\hline Single SVM trained on all training dataset & 0.91 & 100 & 50.45 & - \\
$\begin{array}{l}\text { Single SVM trained on balance training } \\
\text { dataset }\end{array}$ & 69.18 & 53.60 & 61.39 & 0.08 \\
Cascade Classifier & 76.71 & 72.19 & 74.45 & 0.19 \\
\hline
\end{tabular}


Table 4 Comparison between other method and our method based on independent testing dataset

\begin{tabular}{lllll}
\hline & $\operatorname{Sn}(\%)$ & $\mathrm{Sp}(\%)$ & $\mathrm{Acc}(\%)$ & $\mathrm{MCC}$ \\
\hline ASEB & 70.95 & 22.87 & 46.91 & 0.01 \\
GPS-PAIL & 16.41 & 83.12 & 49.77 & -0.003 \\
LAceP & 66.67 & 43.89 & 55.28 & 0.037 \\
PLMLA & 56.76 & 47.38 & 52.07 & 0.015 \\
Our method & 76.71 & 72.19 & 74.45 & 0.19 \\
\hline
\end{tabular}

where $N_{i}$ is the number of alpha helix, beta bridge, strand, helix-3, helix-5, turn or bend and $\mathrm{N}$ is the number of acetylation site or non-acetylation. The result is detailedly shown in Fig. 6.

The frequency of alpha helix on human acetylation sites is less than that on non-acetylation sites, and the frequency of strand on acetylation sites is greater than that on non-acetylation sites, which we can infer that acetylation is more likely to occur in strand region. In addition, obviously, some non-acetylation sites are in beta bridge region while no acetylation sites are beta bridge structure. Based on this phenomenon, we surmise that maybe it is extremely acetylation to happen on beta bridge region. These analyses may offer some new clues for the structural patterns surround the acetylation sites.

Accessible surface area represents the exposed area in protein spatial structure, and biological reaction always happens on the surface of proteins. We statistically calculate the frequency of accessible surface area value in different numerical range of acetylated peptides and non-acetylated peptides, respectively, shown in Fig. 7. As described in Fig. 7, the available surface area values of acetylation sites are concentrated between 60 and 150, and most of the frequency values of acetylation sites in this range are greater than non-acetylation sites. However, non-acetylation sites have advantage in low accessible surface area values, from 0 to 60 , especially between 0 to 10 . We can explain this appearance by reasonable conjecture that the larger the area exposed to the surface, the more likely the acetyl enzyme come into contact it, and if a lysine site is buried in a protein, it will have little chance to take part in the reaction. Therefore, lysine sites with greater accessible surface area are more likely to be acetylated.

\section{Optimal feature selection}

In this study, we employed a two-step feature selection scheme. In the first step, we calculate the mRMR of all features, respectively, and these features are ranked in a list according to fisher-score. Secondly, the first feature is regarded as the basic feature subset and we added features one by one into feature subset from ranked list. In the end, the optimal feature set contains 102 features and the MCC value of different number of features is shown in Fig. 8. Besides, we make a comparison of performance between before feature selection and after feature selection, shown in Table 2. Obviously, not only MCC value, also other performances are improved after feature selection. Besides, the feature dimension is greatly reduced (632 dimensions before feature selection and 102 dimensions after feature selection), which will increase the speed of prediction and save a lot of computational cost.

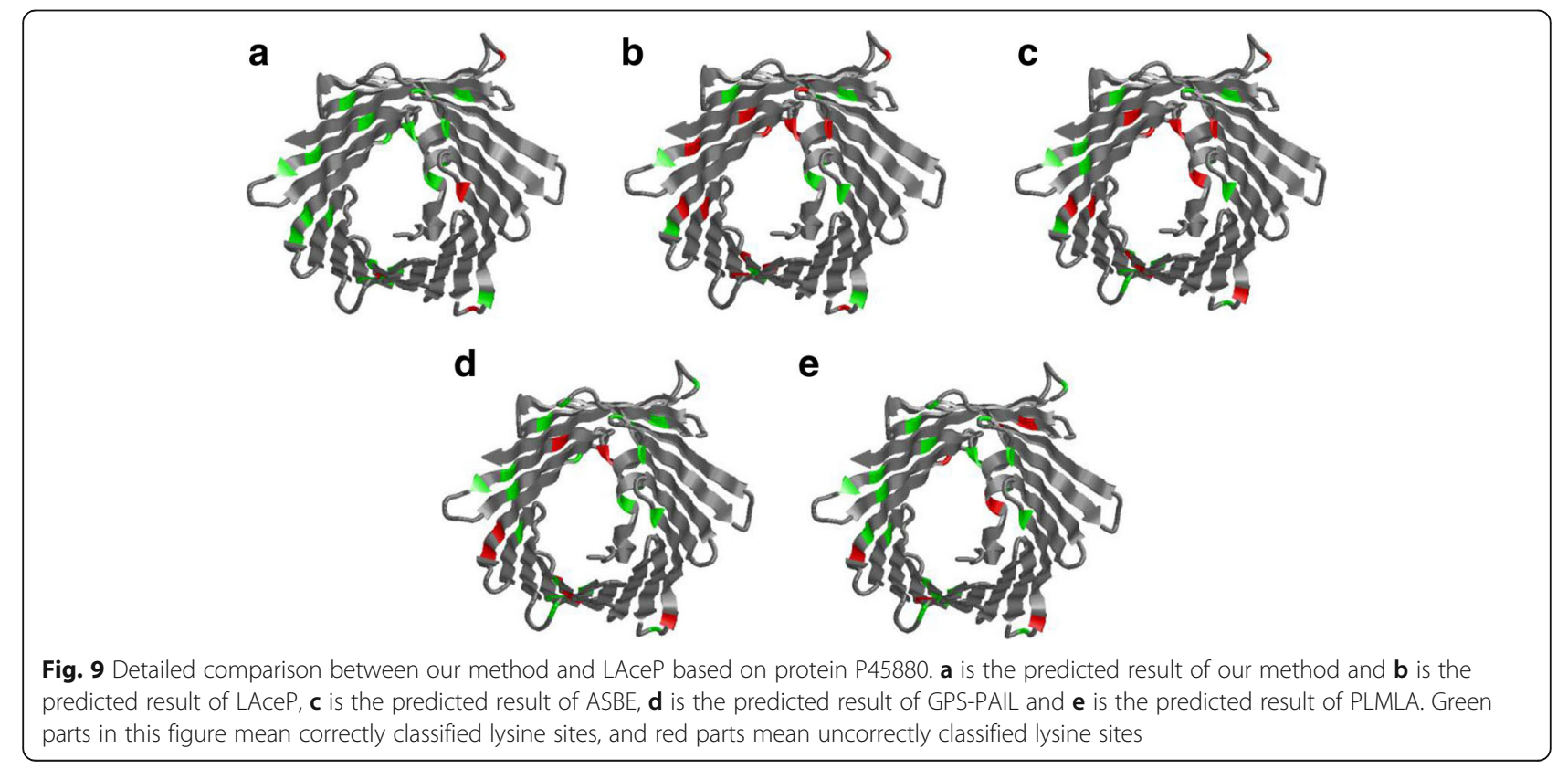


Table 5 Comparison of performances between Homo.sapiens, Mus.musculus and Rattus.norvegicus

\begin{tabular}{lllll}
\hline & $\mathrm{Sn}(\%)$ & $\mathrm{Sp}(\%)$ & $\mathrm{Acc}(\%)$ & $\mathrm{MCC}$ \\
\hline Mus.musculus & 45.78 & 67.91 & 56.85 & 0.089 \\
Rattus.norvegicus & 57.63 & 56.82 & 57.23 & 0.074 \\
Homo.sapiens & 76.71 & 72.19 & 74.45 & 0.19 \\
\hline
\end{tabular}

\section{Cascade classifier result}

In computational methods, most of machine learning algorithms are sensitive to ratio of positive and negative samples. In this study, there are 18,061 non-acetylation sites and 1956 acetylation sites in our training dataset, nearly 10:1 for ratio of negative and positive data, so we construct a cascade classifier based on SVM to solve the imbalance problem between positive data and negative data.

To verify if cascade classifier effectively improved the prediction performances, we compare the performances of cascade classifier and single SVM classifier on independent test dataset, and the results are shown in Table 3. As listed in Table 3, single SVMs always predict a lower Sn value, Acc value and MCC value no matter trained on all training data or trained on balance training dataset. After constructing a cascade classifier based on SVMs, general performance is obviously increased. Single SVM trained on balance training dataset gets a $\mathrm{Sn}$ value that is not too bad, but a relatively poor $\mathrm{Sp}$ value, which may because negative samples used for training are only a part of all negative samples, and contains only partial information. Though Single SVM trained on all training dataset utilizes all negative samples, it results in severe sample imbalance, therefore, the $\mathrm{Sn}$ value is very bad. The cascade classifier not only contains almost all negative sample information, but also effectively solves the problem of sample imbalance, so it gets the best results.

\section{Comparison with exiting methods}

To further evaluate the performance, we compared our method with other published acetylation prediction methods, LAceP [13], PLMLA [9], ASBE [17] and GPSPAIL [4]. Initially, we selected 5 exiting methods to make comparison, but the web server of another method, PSKAcePred [11], can not be used. We put our independent testing dataset on other four methods and obtained the prediction results, shown in Table 4. $\mathrm{Sn}, \mathrm{Sp}$, Acc and MCC are used to measure the performance.

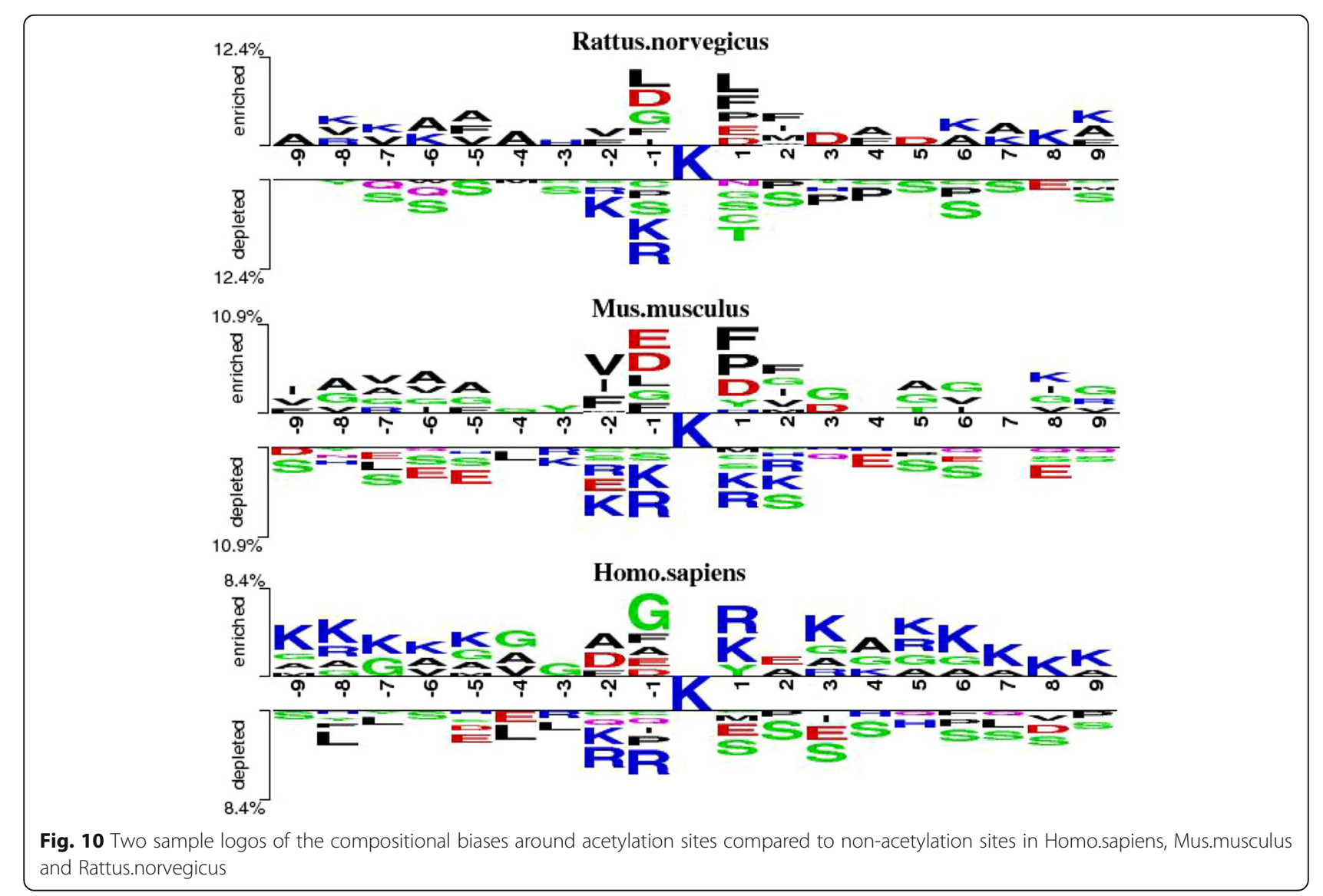


As we can see from Table 4, LAceP get the best performance ( $\mathrm{Sn}$ is $66.67 \%, \mathrm{Sp}$ is $43.89 \%$, Acc is $44.63 \%$ and MCC is 0.037) among ASBE, GPS-PAIL, LAceP and PLMLA, while our method achieve a Sp of $72.19 \%$, a Sn of $76.71 \%$, an Acc of $72.35 \%$ and a MCC of 0.19 , which were much better than other four methods' performance.
Then, we make a detailed comparison of the predicted results on a protein (P45880). Figure 9 describes the specific predicted results, in which green represents the correctly classified lysine sites and red represents the incorrectly classified lysine sites. We can clearly see that green sites occupy a large proportion and we can correctly classified many lysine sites that LAcep incorrectly

\section{a}

\section{Biological Process}

$$
\begin{array}{r}
\text { single-organism cellular process } \\
\text { response to stress } \\
\text { negative regulation of biological process } \\
\text { primary metabolic process } \\
\text { positive regulation of cellular process } \\
\text { organic substance metabolic process } \\
\text { positive regulation of biological process } \\
\text { cellular metabolic process } \\
\text { small molecule metabolic process } \\
\text { single-organism metabolic process }
\end{array}
$$

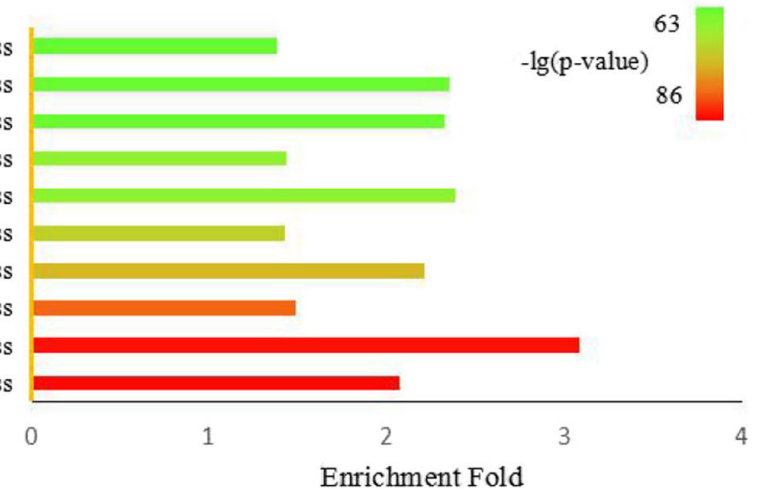

b

Cell Component

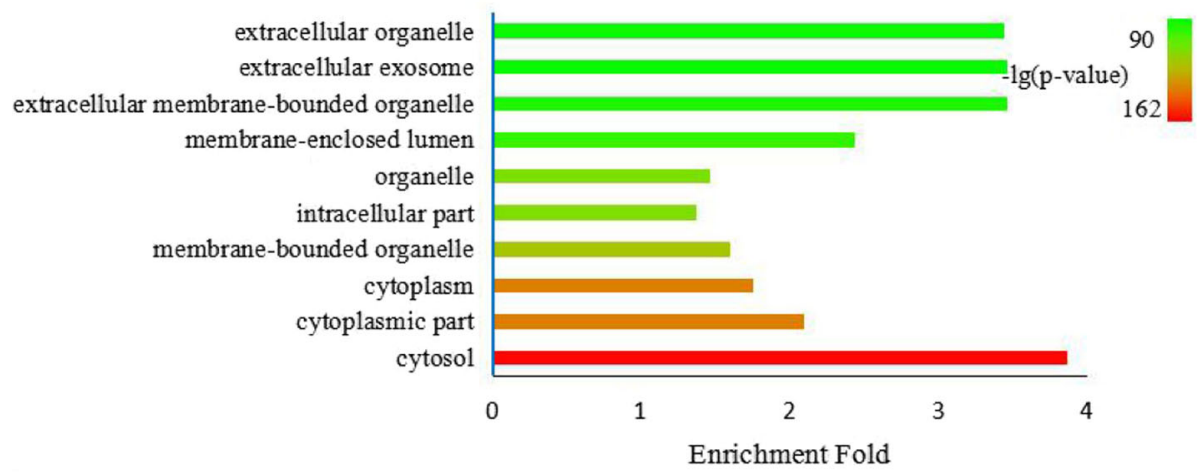

C

Molecular Function

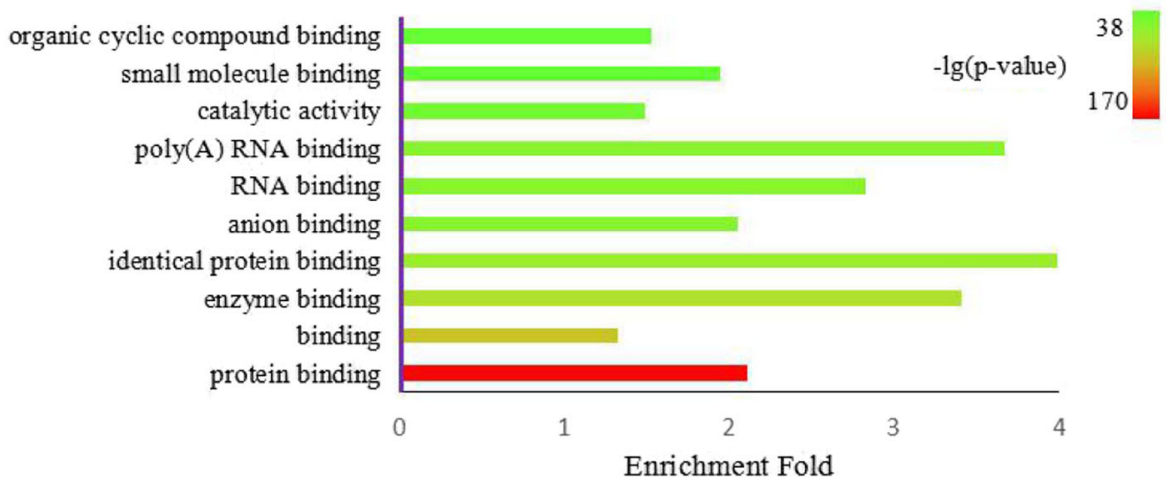

Fig. 11 The top 10 statistically over-represented terms of biological processes, cell component and molecular functions ( $p$-value $<0.01)$. X-axis represents enrichment fold and Y-axis means entries of biological processes, cell component and molecular functions. Different color represents different $p$-value 
classified. Besides, our method also has good prediction accuracy in the helix and sheet structures.

The promising performance and the conclusion from Fig. 9 demonstrate that our method was particularly useful for protein acetylation prediction than other methods.

\section{Comparison between different species}

Due to the specificity among species, different methods should be developed for different species to predict acetylation sites. Our Method is proposed only for prediction of acetylation sites in human proteins. To verify whether our method suit for other species, we selected two mammals to test, because mammals have closer relation with human than prokaryotes or non-mammals. We obtained acetylated proteins of Mus.musculus and Rattus.norvegicus from database mentioned in 2.1, and process data in the same way as section 2.1. Then, we test these two species by our method, and the results are listed in Table 5. From Table 5, we can obviously observe that the performances of Mus.musculus and Rattus.norvegicus are not satisfactory, no matter on Sn, Sp, Acc or MCC.

To explain it, we drew compositional biases around acetylation sites compared to non-acetylation sites in Homo.sapiens, Mus.musculus and Rattus.norvegicus, in Fig. 10. We can see that among the three species, the distribution of amino acids around center lysine is very different, especially in Homo.sapiens and the other two species, which may lead to different mechanisms of lysine acetylation. Therefore, different species may require different methods of classification.

\section{Gene ontology analysis of acetylated proteins}

We statistically analyze the enriched biological processes, cell component and molecular functions with the gene ontology (GO) annotations with Fisher-exact test for acetylated proteins, of which the top 10 statistically significant terms of these three criteria are shown in Fig. 11 ( $p$-value $<0.01)$.

We clearly find that acetylation prefers to occur at diverse metabolic process because among the top 10 biological process, 5 terms are related to metabolic process, including primary metabolic process (GO:0044238), organic substance metabolic process (GO:0071704), cellular metabolic process (GO:0044237), small molecule metabolic process(GO:0044281), single-organism metabolic process (GO:0044710). It has been reported that acetylation may play an important role in the development of cardiovascular diseases through metabolic regulation [54]. Metabolic processes that we found from GO analysis may be entry points for studies on the correlate acetylation with disease. Besides, 3 GO terms are related to regulation, including negative regulation of biological process (GO:0048519), positive regulation of cellular process (GO:0048522), positive regulation of biological process (GO:0048518). Negative regulation of biological process means any process that stops, prevents, or reduces the frequency, rate or extent of a biological process, positive regulation of cellular process any process that activates or increases the frequency, rate or extent of a cellular process, any of those that are carried out at the cellular level, positive regulation of biological process means any process that activates or increases the frequency, rate or extent of a biological process. From this observation, we can infer that acetylation is a key that can active or stop some biological processes.

As for cell component, the top three most significant GO terms are cytosol (GO:0005829), cytoplasmic part (GO:0044444) and cytoplasm (GO:0005737), and intracellular part (GO:0044424) is also in top 10, which are all positions in cellular. And among the top 10 biological process, 3 terms are different extra cellular positions including extracellular membrane-bounded organelle (GO: 0065010), extracellular exosome (GO:0070062) and

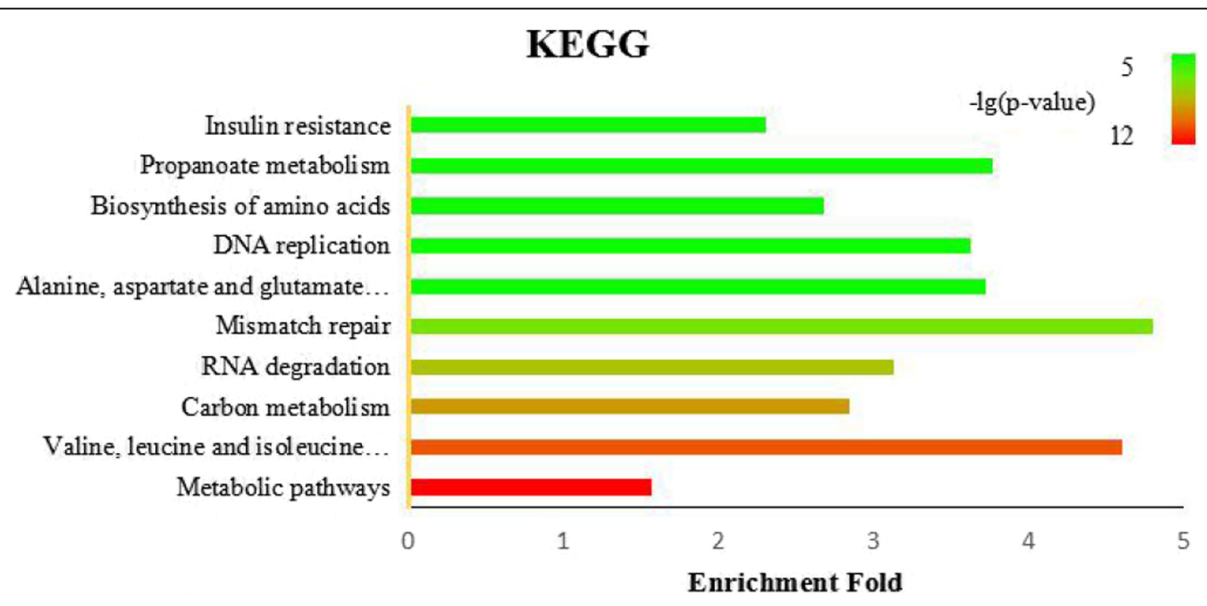

Fig. 12 The enriched KEGG annotations for acetylated proteins ( $p$-value $<0.01$ ). E-fold, Enrichment fold 
extracellular organelle (GO:0043230). These observations indicate that acetylated proteins are active no matter in cellular or out of cellular.

For molecular function, we can obviously find from that 9 terms among the top $10 \mathrm{GO}$ terms are about binding (protein binding (GO:0005515), binding (GO:0005488), enzyme binding (GO:0019899), identical protein binding (GO:0042802), anion binding (GO:0043168), RNA binding (GO:0003723), poly(A) RNA binding (GO:0044822), small molecule binding (GO:0036094), organic cyclic compound binding (GO:0097159)). We can infer from it that acetylation may promote binding between proteins, various ligands and compounds which may cause a lot of diseases. Besides, enzyme have high specificity and catalytic efficiency to their substrates, and catalytic activity (GO: 0003824) means catalysis of a biochemical reaction, both of which are essential for a lot of biological processes and ensure that the intricate biological processes within the cell can proceed in an orderly manner.

Taken together, these observations show that acetylation plays an indispensable role in human body.

\section{KEGG analysis of acetylated protein}

We map all the acetylated protein used in our study to the Kyoto Encyclopedia of Genes and Genomes (KEGG) pathways to further explore functional aspects of acetylation substrates. The top 10 enriched pathways are listed in Fig. $12(p$-value< 0.01$)$ and the statistical result of significant pathways is shown in Fig. 13 ( $p$-value< 0.01$)$.

We can detect that six terms belong to metabolism class, including Metabolic pathways (hsa01100), Valine, leucine and isoleucine degradation (hsa00280), Carbon metabolism (hsa01200), Alanine, aspartate and glutamate metabolism (hsa00250), Biosynthesis of amino acids (hsa01230) and Propanoate metabolism (hsa00640), which is consistent with the result of biological process in GO, meaning that acetylation plays an important role in metabolism. And three terms are subordinate to genetic information processing class, consisting of RNA degradation (hsa03018), Mismatch repair (hsa03430) and DNA replication (hsa03030), and this observation is also clearly emerged form Fig. 13. Except metabolism pathway and genetic information processing pathway, a large portion of acetylated proteins are related to disease. Though organismal system terms and environmental information processing didn't appear in Fig. 12, they occupy a large proportion in Fig. 13. These results concluded demonstrate that acetylation involved in diverse pathways in organism, and the study of acetylation mechanism contributes to the understanding of disease and pharmaceutical industry.

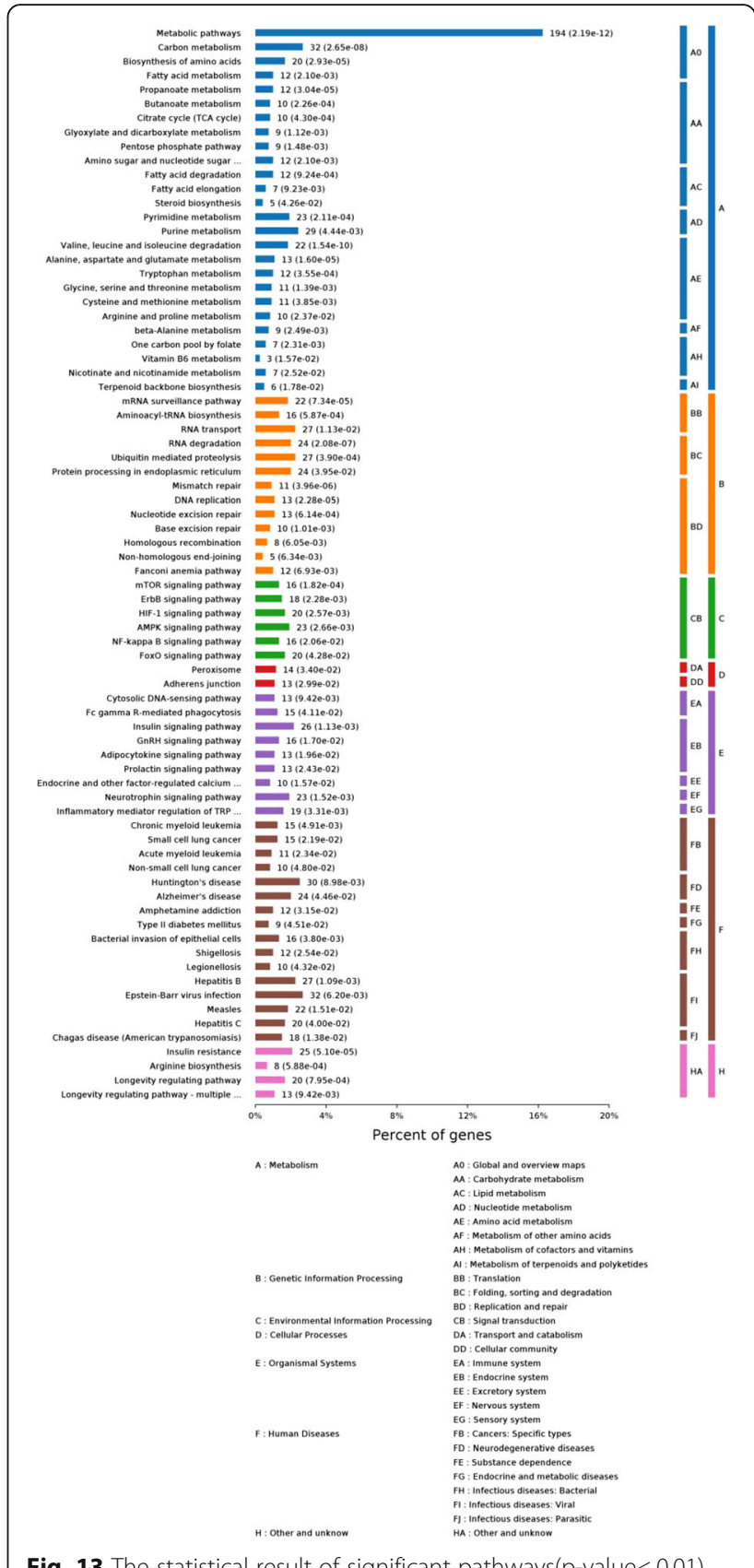

Fig. 13 The statistical result of significant pathways $(p-v a l u e<0.01)$

\section{Conclusions}

In this study, we implement an application of cascade classifier to human protein acetylation prediction problem, combining sequence features and structural features. In this method, we employed a two step feature selection (mRMR and IFS). We proofed that combined feature (sequence features and structural features) is better than sequence feature, and the result of our method is much better than others' which shown that our method is very promising and can be a useful tool to identification of acetylation sites in human. This work 
also indicated that cascade classifier can resolve the imbalance between positive samples and negative samples to improve the performance. We are looking forward that our method will give a powerful help for further studies of acetylation process in human body. We also test that whether different species can get good results on the same method, while the performances of other species are not satisfactory. Therefore, for future work, we are going to seek suitable methods for acetylation sites prediction in other species.

\section{Abbreviations}

AC: auto covariation; Acc: Accuracy; ASA: accessible surface area; GO: Gene Ontology; IE: Information Entropy; IFS: Incremental feature selection; KEGG: Kyoto Encyclopedia of Genes and Genomes; MCC: Matthew's correlation coefficient; mRMR: maximum relevance and minimum redundancy; PCP: physicochemical property; $\mathrm{pH}$ : Hydrogen ion concentration; PSSM: position specific score matrix; PTMs: post-translational modifications; RC: residue composition; Sn: Sensitivity; Sp: Specificity; SS: secondary structure; SVM: Support vector machine

\section{Acknowledgments}

This work was supported by National Natural Science Foundation of China (61403077), the Natural Science Foundation for Youths of JiLin Province (20150520061JH), the Fundamental Research Funds for the Central Universities (2412019FZ047)

\section{Authors' contributions}

ZM, XZ and QN conceived and designed the experiments. QN performed the experiments. JJ and MY collected the data. QN wrote the manuscript with revision by XZ. All authors read and approved the final manuscript.

\section{Authors' information}

Qiao Ning is a PhD candidate in Northeast Normal University. Her main interest includes prediction of PTM sites.

Miao Yu is a graduate student in Northeast Normal University. Her main interest includes prediction of PTM sites.

Jinchao $\mathrm{Ji}$ is a teacher in Northeast Normal University. His main interest includes machine learning and data mining.

Zhiqiang Ma is a professor in Northeast Normal University. His main interest includes prediction of PTM sites and fuctional sites.

Xiaowei Zhao is a teacher in Northeast Normal University. Her main interest includes prediction of PTM sites and artificial intelligence algorithm.

\section{Funding}

This work was supported by National Natural Science Foundation of China (61403077), the Natural Science Foundation for Youths of JiLin Province (20150520061JH), the Fundamental Research Funds for the Central Universities (2412019FZ047), which fund us to build the environment we needed for our experiments, including facilities for data collection and analysis.

\section{Availability of data and materials}

All data used in our study can be downloaded from the http://plmd. biocuckoo.org/, http://cplm.biocuckoo.org/, https://www.phosphosite.org/ homeAction.action, https://www.uniprot.org/, http://www.rcsb.org/.

\section{Ethics approval and consent to participate}

Not applicable.

\section{Consent for publication}

Not applicable.

\section{Competing interests}

The authors declare that they have no competing interests.
Received: 31 March 2019 Accepted: 6 June 2019

Published online: 17 June 2019

\section{References}

1. Machida Y, Chiba T, Takayanagi A, et al. Common anti-apoptotic roles of parkin and alpha-synuclein in human dopaminergic cells.[J]. Biochemical \& Biophysical Research Communications. 2005;332(1):233-40.

2. Lind C, Gerdes R, Hamnell Y, et al. Identification of S-glutathionylated cellular proteins during oxidative stress and constitutive metabolism by affinity purification and proteomic analysis.[J]. Archives of Biochemistry \& Biophysics. 2002:406(2):229-40.

3. Lu CT, Lee TY, Chen YJ, et al. An intelligent system for identifying acetylated lysine on histones and nonhistone proteins.[J]. Biomed Res Int. 2015; 2014(2014):528650.

4. Deng W, Wang C, Zhang Y, et al. GPS-PAlL: prediction of lysine acetyltransferase-specific modification sites from protein sequences[]]. Sci Rep. 2016;6:39787.

5. Li S, Li H, Li M, et al. Improved prediction of lysine acetylation by support vector machines.[J]. Protein Pept Lett. 2009;16(8).

6. Lee TY, Hsu BK, Lin FM, et al. N-ace: using solvent accessibility and physicochemical properties to identify protein $\mathrm{N}$-acetylation sites[]]. J Comput Chem. 2010;31(15):2759-71.

7. $\mathrm{Xu} Y$, Wang $\mathrm{XB}$, Ding J, et al. Lysine acetylation sites prediction using an ensemble of support vector machine classifiers[]]. J Theor Biol. 2010;264(1): 130-5.

8. Shao J, Xu D, Hu L, et al. Systematic analysis of human lysine acetylation proteins and accurate prediction of human lysine acetylation through birelative adapted binomial score Bayes feature representation.[J]. Mol BioSyst. 2012;8(11):2964-73.

9. Shi SP, Qiu JD, Sun XY, et al. PLMLA: prediction of lysine methylation and lysine acetylation by combining multiple features.[J]. Mol BioSyst. 2012;8(5): $1520-7$.

10. Shi SP, Qiu JD, Sun XY, et al. A method to distinguish between lysine acetylation and lysine methylation from protein sequences[]]. J Theor Biol. 2012;310(4):223-30

11. Suo SB, Qiu JD, Shi SP, et al. Position-specific analysis and prediction for protein lysine acetylation based on multiple features[J]. PLoS One. 2012; 7(11):e49108.

12. Suo SB, Qiu JD, Shi SP, et al. Proteome-wide analysis of amino acid variations that influence protein lysine acetylation[J]. J Proteome Res. 2013; 12(2):949-58.

13. Hou $T$, Zheng $G$, Zhang $P$, et al. LAceP: lysine acetylation site prediction using logistic regression classifiers[J]. PLoS One. 2014;9(2):e89575.

14. Li Y, Wang M, Wang $\mathrm{H}$, et al. Accurate in silico identification of speciesspecific acetylation sites by integrating protein sequence-derived and functional features[]]. Sci Rep. 2014;4(无):5765.

15. Qiu WR, Sun $B Q$, Xiao X, et al. iPTM-mLys: identifying multiple lysine PTM sites and their different types[]]. Bioinformatics. 2016;32(20):3116-23.

16. Qiqige W, Wei Z, Zhang Y, et al. Improved species-specific lysine acetylation site prediction based on a large variety of features set:[]]. PLoS One. 2016; 11(5):e0155370.

17. Wang L, Du Y, Lu M, et al. ASEB: a web server for KAT-specific acetylation site prediction.[J]. Nucleic Acids Res. 2012;40(Web Server issue):376-9.

18. Liu Z, Wang Y, Gao T, et al. CPLM: a database of protein lysine modifications[]]. Nucleic Acids Res. 2014;42:Database issue):531-6.

19. $\mathrm{Xu} \mathrm{H}$, Zhou J, Lin S, et al. PLMD: an updated data resource of protein lysine modifications[]]. Journal of genetics and genomics $=Y i$ chuan $x$ ue bao. 2017:44(5):243-50.

20. Hornbeck PV, Kornhauser JM, Tkachev S, et al. PhosphoSitePlus: a comprehensive resource for investigating the structure and function of experimentally determined post-translational modifications in man and mouse[J]. Nucleic Acids Res. 2012;40(Database issue):D261-70.

21. Boutet $E$, Lieberherr $\mathrm{D}$, Tognolli $\mathrm{M}$, et al. UniProtKB/Swiss-Prot, the manually annotated section of the UniProt KnowledgeBase: how to use the entry view[J]. Methods Mol Biol. 2016;1374:23-54.

22. Westbrook J, Feng Z, Jain $\mathrm{S}$, et al. The protein data Bank: unifying the archive[J]. Nucleic Acids Res. 2002;30(1):245.

23. Peri S, Navarro JD, Amanchy $R$, et al. Development of human protein reference database as an initial platform for approaching systems biology in humans.[J]. Genome Res. 2003;13(10):2363. 
24. Hu L L, Li Z, Wang $K$, et al. Prediction and analysis of protein methylarginine and methyllysine based on multisequence features[J]. Biopolymers, 2011, 95(11):0-0.

25. Zhao X, Li X, Ma Z, et al. Prediction of lysine Ubiquitylation with ensemble classifier and feature selection[J]. Int J Mol Sci. 2011;12(12):8347-61.

26. Kawashima S, Ogata H, Kanehisa M. AAindex: amino acid index database.[J]. Nucleic Acids Res. 1999;27(1):368-9.

27. Zhao X, Ning Q, Ai M, et al. PGluS: prediction of protein S-glutathionylation sites with multiple features and analysis[J]. Mol BioSyst.

28. Yutani K, Ogasahara K, Tsujita T, et al. Dependence of conformational stability on hydrophobicity of the amino acid residue in a series of variant proteins substituted at a unique position of tryptophan synthase alpha subunit.[J]. Proc Natl Acad Sci U S A. 1987;84(13): 4441-4.

29. Vihinen M, Torkkila E, Riikonen P. Accuracy of protein flexibility predictions. [J]. Proteins-structure Function \& Bioinformatics. 2010;19(2):141-9.

30. Monné $M$, Hermansson $M$, Von HG. A turn propensity scale for transmembrane helices.[J]. J Mol Biol. 1999;288(1):141-5.

31. Altschul SF, Madden TL, Schaffer AA, et al. Gapped BLAST and PSI-BLAST: a new generation of protein database search programs. Nucleic Acids Res. 1997;25(17):3389-402.

32. Yu D, Shen H, Yang J. SOMRuler: a novel interpretable transmembrane helices predictor[J]. IEEE Trans Nanobioscience. 2011;10(2):121-9.

33. Yu DJ, Shen HB. SOMPNN: an efficient non-parametric model for predicting transmembrane helices[J]. Amino Acids. 2012:42(6):2195-205.

34. Zangooei MH, Jalili S. Protein secondary structure prediction using DWKF based on SVR-NSGAII[J]. Neurocomputing. 2012;94(3):87-101.

35. Zhang Y N, Yu D J, Li S S, et al. Predicting protein-ATP binding sites from primary sequence through fusing bi-profile sampling of multi-view features[J]. Bmc Bioinformatics, 2012, 13(1):118-118.

36. Dong Q, Zhou S, Guan J. A new taxonomy-based protein fold recognition approach based on autocross-covariance transformation[J]. Bioinformatics. 2009;25(20):2655-62

37. Guo Y, Yu L, Wen Z, et al. Using support vector machine combined with auto covariance to predict protein-protein interactions from protein sequences[J]. Nucleic Acids Res. 2008;36(9):3025-30.

38. Liu $B$, Wang $X$, Lin $L$, et al. A discriminative method for protein remote homology detection and fold recognition combining top$\mathrm{n}$-grams and latent semantic analysis[J]. Bmc Bioinformatics, 2008 9(1):510-510.

39. Landreh M, Astorga-Wells J, Johansson J, et al. New developments in protein structure-function analysis by MS and use of hydrogen-deuterium exchange microfluidics[J]. FEBS J. 2011;278(20):3815-21.

40. Kabsch W, Sander C. Dictionary of protein secondary structure: pattern recognition of hydrogen-bonded and geometrical features[J]. Biopolymers. 2010;22(12):2577-637.

41. Ehrlich $L$, et al. Prediction of waterbinding sites on proteins using neural networks. Protein Eng. 1998;11:11-9.

42. Chou KC. Prediction of signal peptides using scaled window.[J]. Peptides. 2001;22(12):1973-9.

43. Chen W, Feng PM, Lin H, et al. IRSpot-PseDNC: identify recombination spots with pseudo dinucleotide composition[J]. Nucleic Acids Res. 2013; 41(6):e68.

44. Qiu WR, Jiang SY, Sun BQ, et al. iRNA-2methyl: identify RNA 2'-Omethylation sites by incorporating sequence-coupled effects into general PseKNC and ensemble classifier.[J]. Med Chem. 2017;13(8).

45. Qiu WR, Jiang SY, Xu ZC, et al. iRNAm5C-PseDNC: identifying RNA 5methylcytosine sites by incorporating physical-chemical properties into pseudo dinucleotide composition[J]. Oncotarget. 2017:8(25): 41178-88.

46. Shahid A, Maqsood H. iMethyl-STTNC: identification of $\mathrm{N} 6$ -methyladenosine sites by extending the idea of SAAC into Chou's PseAAC to formulate RNA sequences[J]. J Theor Biol. 2018:S0022519318303436.

47. Wei C, Hui D, Xu Z, et al. iRNA(m6A)-PseDNC: identifying N6methyladenosine sites using pseudo dinucleotide composition[J]. Anal Biochem. 2018:S0003269718307632

48. Chen W, Feng $\mathrm{P}$, Yang $\mathrm{H}$, et al. iRNA-3typeA: identifying three types of modification at RNA's adenosine sites.[J]. Molecular Therapy Nucleic Acids. 2018;11:468-74.
49. Feng $P$, Yang $H$, Ding $H$, et al. iDNA6mA-PseKNC: identifying DNA N 6 -methyladenosine sites by incorporating nucleotide physicochemical properties into PseKNC[J]. Genomics. 2018:S0888754318300090.

50. Peng $\mathrm{HC}$, Long $\mathrm{FH}$, Ding $\mathrm{C}$. Feature selection based on mutual information: criteria of max-dependency, max-relevance, and min-redundancy. leee $T$ Pattern Anal. 2005;27(8):1226-38.

51. Wiley WN. Statistical learning theory. New York: John Wiley; 1998.

52. Chang CC, Lin CJ. LIBSVM: a library for support vector machines. ACM T INTEL SYST TEC. 2011;2(3):27.

53. Wei ZS, Yang JY, Shen HB, et al. A Cascade random forests algorithm for predicting protein-protein interaction sites[J]. IEEE Transactions on Nanobioscience. 2015;14(7):746-60

54. Yiping $W$. Regulation of metabolism by lysine acetylation and its role in metabolic diseases[J]. Sci Sinica. 2015;45(11):1083-92

\section{Publisher's Note}

Springer Nature remains neutral with regard to jurisdictional claims in published maps and institutional affiliations.
Ready to submit your research? Choose BMC and benefit from:

- fast, convenient online submission

- thorough peer review by experienced researchers in your field

- rapid publication on acceptance

- support for research data, including large and complex data types

- gold Open Access which fosters wider collaboration and increased citations

- maximum visibility for your research: over $100 \mathrm{M}$ website views per year

At $\mathrm{BMC}$, research is always in progress.

Learn more biomedcentral.com/submissions 